\title{
Strain path change effect on dislocation microstructure of multicrystalline copper sheets
}

\author{
N.A. Sakharova*, J.V. Fernandes \\ Departamento de Engenharia Mecânica-FCTUC, Pólo 2, Universidade de Coimbra, CEMUC, Pinhal de Marrocos, P-3030-201 Coimbra, Portugal
}

Received 1 September 2005; received in revised form 13 December 2005; accepted 27 January 2006

\begin{abstract}
In this study, coarse-grained copper sheets were subjected to tension-rolling and rolling-tension strain path sequences. In both cases, two different types of strain path change were studied: the tensile and rolling directions were parallel and normal to each other. TEM observations of deformed samples showed the typical dislocation structures for the prestraining paths in tension and rolling. Special microband features, not observed during prestrain, were found during the second strain path, whatever the sequence and type of strain path change. The microstructure observed during reloading is discussed in terms of the sequence and type of strain path change, parallel or normal. The frequency of appearance of microbands is discussed in terms of the activity of new slip systems, i.e. not active during the prestrain path and connected with the number of the active slip systems after reloading. The results from this study, obtained for coarse-grained multicrystalline copper sheets, are compared with previous ones for fine and medium-grained copper.
\end{abstract}

(c) 2006 Elsevier B.V. All rights reserved.

Keywords: Metals; Mechanical testing; Electron microscopy; Microstructure

\section{Introduction}

Microstructure evolution in fine or medium-grained copper subjected to plastic deformation has been substantially investigated during recent years [1-7]. It was found that microstructure evolution leads to the formation of low-energy dislocation structures. Investigation into the dislocation structures formed during tensile straining of copper (grain size 20 and $250 \mu \mathrm{m}$ ) revealed three types of dislocation microstructures depending on the grain orientation [2,7]: (i) one set of dislocation walls parallel to the main active slip plane; (ii) two sets of extended dislocation straight walls parallel to the two main active slip planes; (iii) equiaxed cell structure, when three or more slip systems have similar activity. It has been shown that the structural morphology of the dislocation walls is similar in the fine and medium grains.

The majority of the performed studies concern monotonous strain path, although strain path change, which frequently arises during sheet metal forming, can significantly influence the microstructural evolution of the material. For this reason,

\footnotetext{
* Corresponding author. Tel.: +351 239 790700; fax: +351 239790701

E-mail address: nataliya.sakharova@dem.uc.pt (N.A. Sakharova).
}

a combination of several simple loading test sequences is an effective way to investigate the dislocation microstructure of sheet metals under such real forming conditions. Several studies connected with dislocation evolution during complex strain paths deal with sequences of two uniaxial tension tests or orthogonal rolling-tension $[1-4,7,8]$. The influence of the dislocation microstructure developed during the first strain path on the behaviour after reloading in the second path, was understood in terms of the interaction between the mobile dislocations in the new active slip systems and the dislocation microstructure previously formed during prestraining. In these studies, special microband features caused by intense glide on one slip plane were observed after strain path change, at relatively low deformation values. These microbands correspond to regions of the crystal with a misoriented crystal lattice, and have one dimension, which is smaller than the two others. They have also been observed at large strain values during monotonic plastic deformation, in a wide range of metallic materials $[3,4,6,9,10]$. These microbands can play an important role in the deformation process because of their ability to accumulate large shear strains. In the case of strain path change, these special microband features were detected at lower strain values than in simple rolled copper and the appearance of such microbands depends on the grain size and, consequently, 
depends on the strain accommodation processes between grains [3,7]. From this point of view, the investigation of the appearance and development of special microband features in coarse-grained copper samples is worthy of interest.

This study is devoted to the investigation of the microstructural evolution of coarse-grained multicrystal copper sheets during complex strain paths. These multicrystalline samples are only a few-grain thick. The use of multicrystalline materials to study plastic deformation mechanisms gives a better understanding of the plastic deformation behaviour of polycrystals. This is because of the absence of constraint in the direction perpendicular to the sample surface, and therefore the strain accommodation processes between grains are insignificant, which clarifies the analysis. In this study the microstructure evolution of multicrystalline copper sheets was investigated for tension-rolling and rolling-tension sequences, the second path of each sequence being such that the tensile and the rolling directions are parallel and normal to each other.

\section{Experimental procedures}

The material used was oxygen free high purity copper (DHP) with a purity of $99.95 \%$. The samples were cut from a cold-rolled and annealed sheet, $1 \mathrm{~mm}$ thick and with an average grain size of $20 \mu \mathrm{m}$, and then were annealed for $20 \mathrm{~h}$ at $T=925^{\circ} \mathrm{C}$ in a $10^{-5} \mathrm{mbar}$ vacuum in order to obtain a structure with a mean grain size in the sheet surface of about $1.5 \mathrm{~mm}$, and showing between 1 and 3 grains in thickness. The samples obtained were deformed by means of four types of complex strain paths, using the sequences: tension-rolling and rolling-tension. Two different types of strain path change were studied for both sequences: the tensile and rolling directions were parallel and normal to each other, in the sheet plane. Specimens for the tension-rolling sequence were prestrained in tension up to moderate strain values (lower than 25\%) and then were rolled parallel and normal to the previous tensile direction, up to strain amounts lower than $20 \%$. For the rolling-tension sequence, the prestrained specimens measured $100 \mathrm{~mm} \times 100 \mathrm{~mm}$ and the tensile samples had a gauge size of $60 \mathrm{~mm} \times 10 \mathrm{~mm}$. The samples for the rolling-tension sequence were deformed up to $5 \%$ of tensile deformation (necking occurs at this deformation value) after prestraining in rolling up to $20 \%$. All tensile tests were performed at an initial strain rate of $10^{-4} \mathrm{~s}^{-1}$.

Observation of microstructure after deformation was carried out using both optical and transmission electron microscopy. The observation plane was the sheet plane, for both cases. Slip lines were observed in the tensile samples after $10 \%$ of deformation by the interferential contrast technique in the optical microscope. At first, the samples for optical observation was deformed up to $5 \%$ and then polished and deformed again up to $10 \%$ in simple tension. These samples were polished using a solution containing $250 \mathrm{ml}$ orthophosphoric acid, $250 \mathrm{ml}$ ethanol, $500 \mathrm{ml}$ distilled water and $3 \mathrm{~g}$ urea and using a current density of $60 \mathrm{~A} \mathrm{dm}^{-2}$. The dislocation microstructure was studied by TEM. Samples for TEM were polished mechanically from both sides down to a thickness of about $0.15 \mathrm{~mm}$ and then electropolished using a double jet thinner. A dilute solution of orthophosphoric acid (2:1) at room temperature under $10 \mathrm{~V}$ tension was used. TEM analysis was carried out at $100 \mathrm{kV}$.

\section{Results and discussion}

Analyses of deformation microstructure formed in the samples were carried out on two scales: surface characterization of slip lines, by optical microscopy and dislocation microstructure, by TEM. Also the mechanical behaviour in tension parallel and normal to previous rolling was studied for comparison.

\subsection{Slip lines analyses}

Slip line observations were performed on the sheet plane in order to reveal the development of active slip systems during monotonic tension testing. Fig. 1 shows two examples of surface slip trace observations on the copper multicrystalline sample. Most grains present one family of parallel slip lines. In some cases, the slip lines are aligned along two main directions in the same grain. This can occur, for example, when structural twins are present: the slip line families, situated along different main directions, are separated by the twin boundary.

In conclusion, optical observations of slip line show, for copper multicrystalline sheets with coarse grain size strained in tension up to $10 \%$ of deformation, dislocation gliding mainly on one active slip system, whatever the region of the grain, which is similar with results obtained for large grain size copper polycrystals $(250 \mu \mathrm{m})$ [2]. These results allow us to confirm on a larger scale (several grains) TEM observations of the dislocation microstructure discussed in the next paragraph. Indeed, such observations are restricted to limited regions inside each grain, taking into account the relatively large size of the grains.

\subsection{Dislocation microstructure}

Firstly, the dislocation microstructures developed during prestraining paths, simple tension and simple rolling, were analyzed. Depending on the grain orientation, two main types of microstructure were found in the samples prestrained in pure tension up to $25 \%$ of deformation: (i) an unorganized

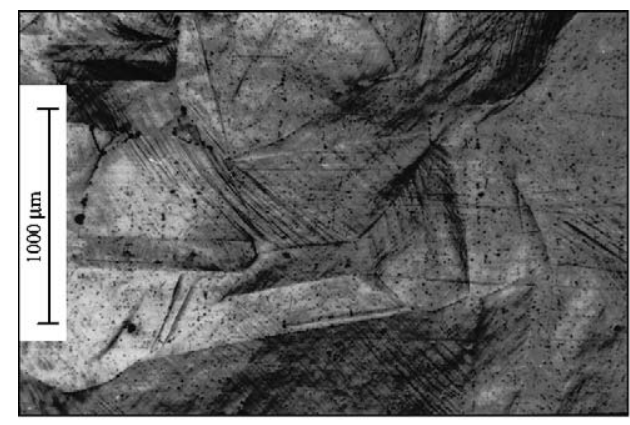

(a)

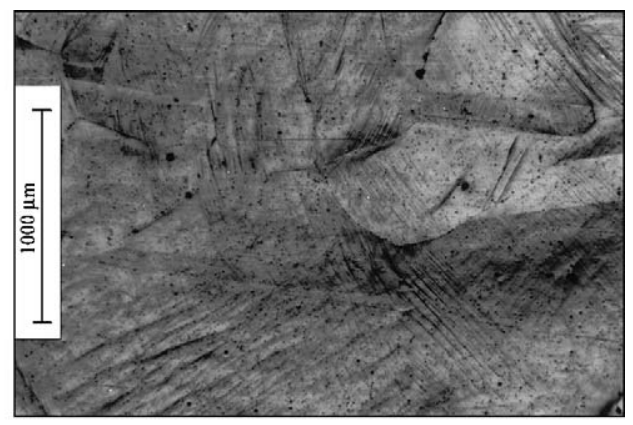

(b)

Fig. 1. Examples of slip line observations in the copper multicrystalline sheet, after $10 \%$ of deformation in pure tension (tensile direction: horizontal) 


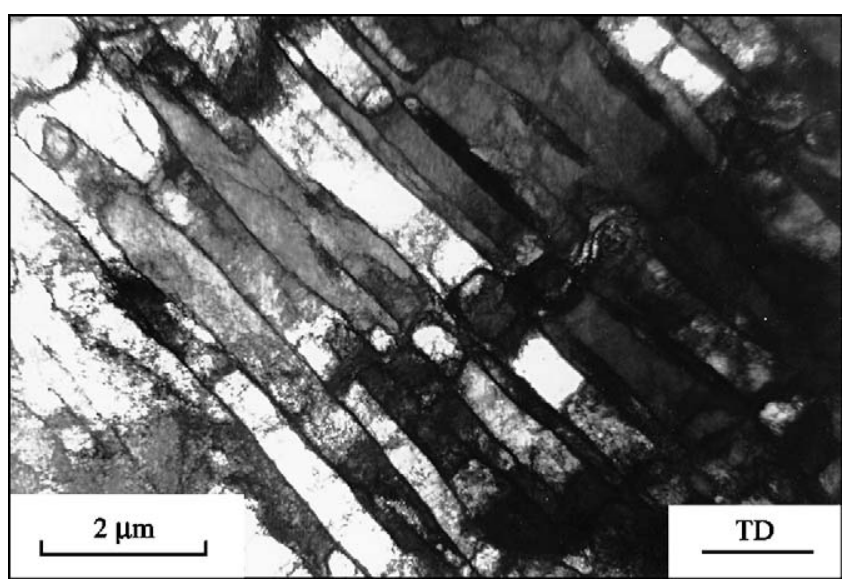

Fig. 2. Parallel dislocation walls in copper multicrystal subjected to $25 \%$ of simple tension deformation (TD: tensile direction).

dislocation structure, i.e. cell walls not yet developed and (ii) one family of dislocation walls closely parallel to the traces of a $\left\{\begin{array}{lll}1 & 1 & 1\end{array}\right\}$ plane (Fig. 2). The first case indicates that the density of dislocations is relatively low for this amount of deformation. Also, the observed walls are less organized than in the cases of fine and even medium-grained copper polycrystals (from 20 to $250 \mu \mathrm{m}[1,2])$, at the same strain value in tension. This kind of structure indicates the main activity of only one slip system up to $25 \%$ of deformation, as confirmed by slip line observations during simple tension (see above). The activity of only one slip system and the low dislocation density is consistent with the fact that the strain accommodation processes between grains are eventually limited to few regions near the grain boundary for large grain sizes, and in particular for this example of coarsegrained multicrystals deformed in tension. In conclusion, these results concerning the dislocation microstructure developed in tension are similar to those obtained for single crystals.

After $20 \%$ prestraining in pure rolling, three types of dislocation configurations were observed. One of them contains equiaxed cells and occurs frequently (Fig. 3). These equiaxed cells are not well organized, their average size being about 1.0-1.5 $\mu \mathrm{m}$. The cell walls consist of tangled dislocation lines

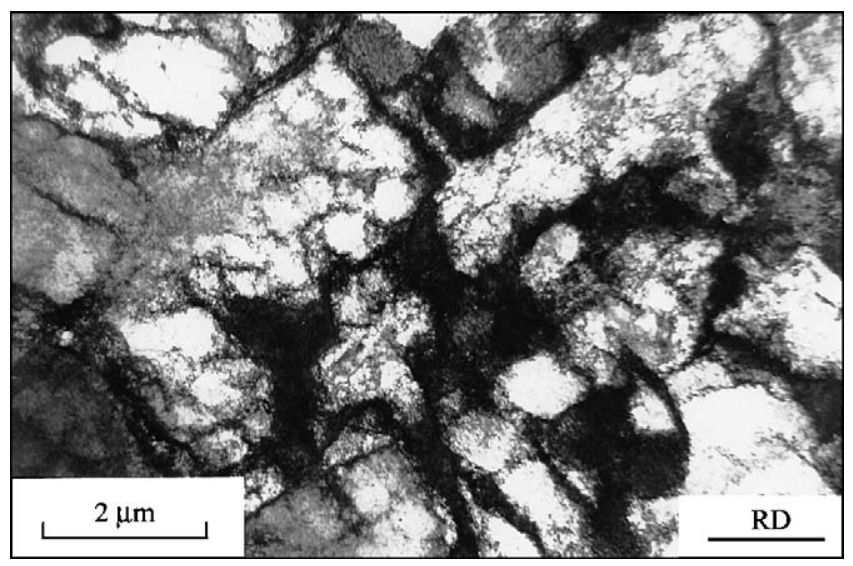

Fig. 3. Equiaxed cells in copper multicrystal subjected to $20 \%$ of simple rolling deformation (RD: rolling direction). and have a width close to $0.05-0.3 \mu \mathrm{m}$. The interior cell areas are almost free of dislocations. Equiaxed cells are quite typical of rolling deformation of fine and medium-grained copper [1-3]. A second dislocation feature observed in pure rolling consists of two sets of parallel dislocation walls crossing each other. Finally, some grains contain only one family of long straight parallel dislocation walls and in some places short walls appear between them, forming a cellblock structure. These straight walls are parallel to the traces of $\left\{\begin{array}{lll}1 & 1 & 1\end{array}\right\}$ planes, as in pure tension. As was previously discussed [2], the shape of the dislocation cells is related to the number of active slip systems. The appearance of equiaxed cells indicates the presence of three or more main active slip planes and each long straight wall corresponds to one main active slip plane. Actually, pure rolling deformation is more constrained than tension and requires a larger number of active slip systems for most grain orientations, independently of the accommodation between grains. This is because, in pure rolling, the multicrystalline sheet and, consequently, the grains cannot deform in the direction of the rolling cylinders. In addition, rolling leads to frictional stresses on the sheet surface; this may also influence the deformation character of the material, originating dislocation structures which are different from those caused by pure tension. This fact is confirmed by the observed microstructures in rolling which indicate activation of several (one, two or more) main slip systems, depending on grain orientation, although the strain accommodation processes between grains have to be insignificant, for such large grains.

The four types of sequential strain paths investigated in the present paper were chosen in order to understand the influence of the magnitude of the change of strain path, normal and parallel, on the microstructure developed after reloading in tension and rolling. The magnitude of the change of strain path can be quantified by a parameter $\alpha$, previously proposed by Schmitt et al. [11], which is defined as the tensorial product of the two successive strain tensors divided by the norm of each tensor, i.e. corresponds to the cosine of the angle between the two vectors which represent the successive strain tensors. This parameter is equal to zero for the present orthogonal strain path changes and equal to 0.87 for the cases of parallel strain path changes. The value of $\alpha$ close to zero indicates severity of strain path change, connected with the fact that most of the active slip systems during the reloading have been inactive during the prestrain [12]. This is, in many circumstances, a condition for the development of structural instabilities in the form of microbands [3,7]. A value of $\alpha$ close to 1 , for which no changes in strain path occurs, such as $\alpha$ equal to 0.87 , indicates a smooth strain path change, without significant activation of new slip systems, i.e. not active during prestrain [12].

Concerning the results of complex strain paths, the rolling-tension sequence will be analysed first. For both types of strain path change studied, tension parallel and normal to previous rolling direction, equiaxed cell structures and two sets of long dislocation walls were observed (Figs. 4 and 5). These dislocation walls were partially dissolved and in some cases became wavy; coarse rectangular cells, larger than $1 \mu \mathrm{m}$, were formed by intersected walls. These structures were not observed in simple tension, but they are typical for pure rolling deformation. This 


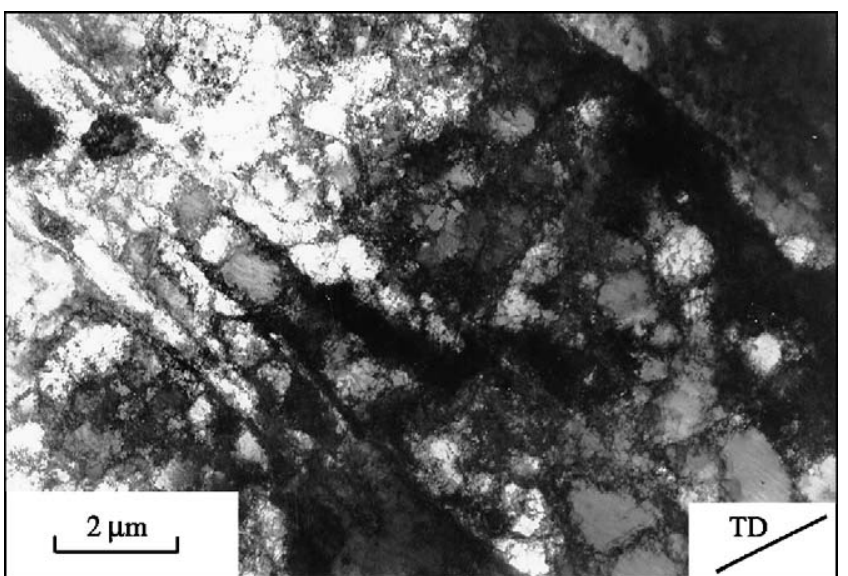

Fig. 4. Equiaxed cells developed in the sequence rolling (20\%)-tension (5\%), tension is normal to rolling (TD: tensile direction).

indicates that $5 \%$ of deformation in second path in tension was not enough to destroy the previous dislocation structure formed in rolling. The most characteristic structural features arising in copper multicrystals deformed in tension after rolling are isolated thin microbands aligned with the trace of $\left\{\begin{array}{lll}1 & 1 & 1\end{array}\right\}$ planes (Fig. 6). These isolated microbands cross all grain areas and appear frequently in investigated grains, whatever the change of strain path, normal and parallel. Nevertheless, microbands are more frequent when the direction of the second strain path in tension is normal to the previous rolling that corresponds to a

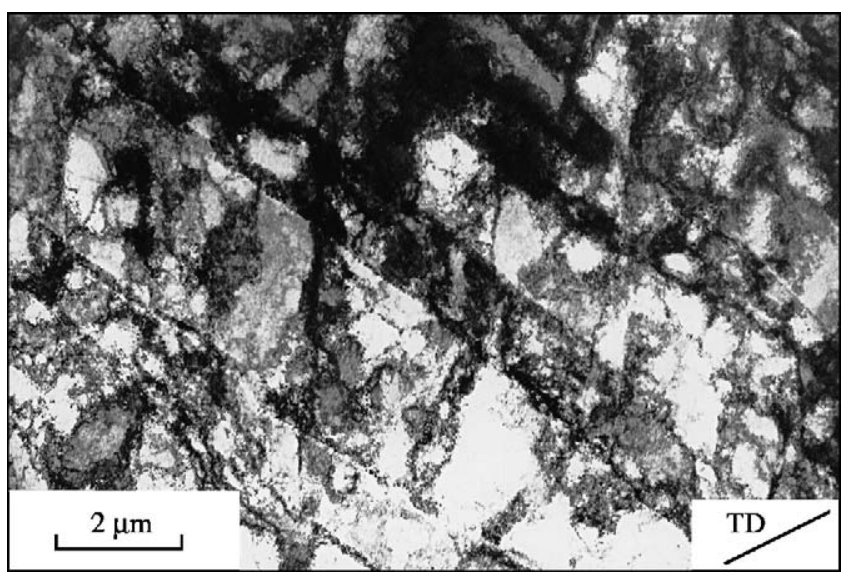

Fig. 5. Two sets of dislocation walls developed in the sequence rolling (20\%)-tension (5\%), tension is parallel to rolling (TD: tensile direction).

severe change of strain path: $\alpha$ is equal to zero. Fig. 7 shows the fraction of grains, $F$, with different kinds of microstructure in tension after rolling, when tensile direction is normal (Fig. 7a) and parallel (Fig. 7b) to the previous rolling direction. A number of 40 individual grains were observed, for each case of strain path change, parallel and normal.

As has been investigated previously [3,7], such special microband features are developed by intense gliding on just one slip plane after strain path change, when a previous dislocation structure has already been formed. This is compatible with strain

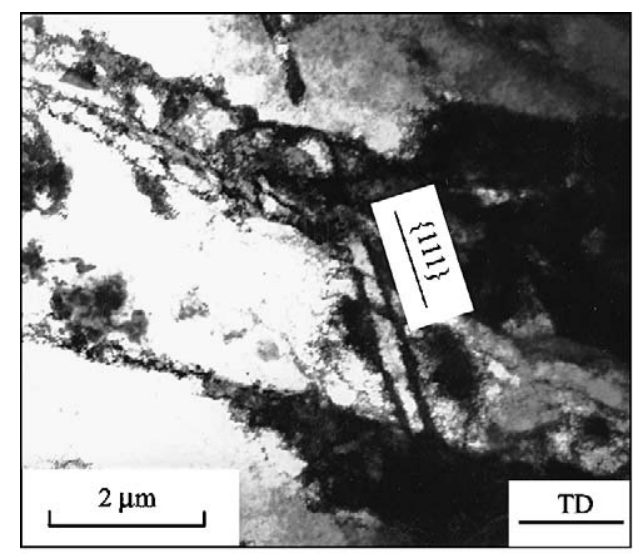

(a)

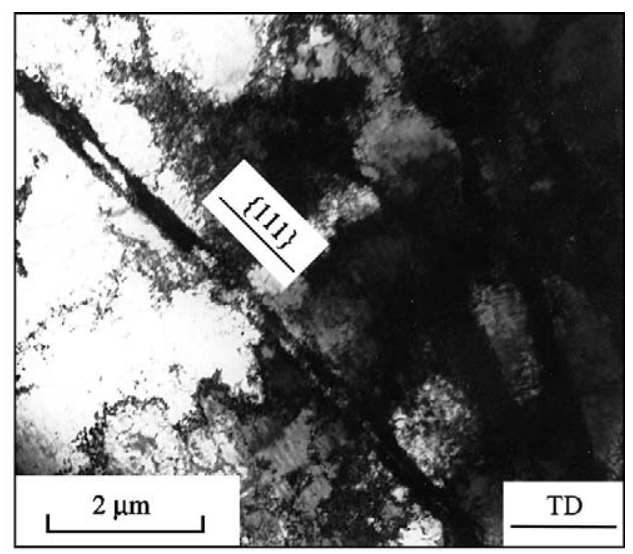

(b)

Fig. 6. Microbands developed in the sequence rolling (20\%)-tension (5\%), tension is normal to rolling (TD: tensile direction).
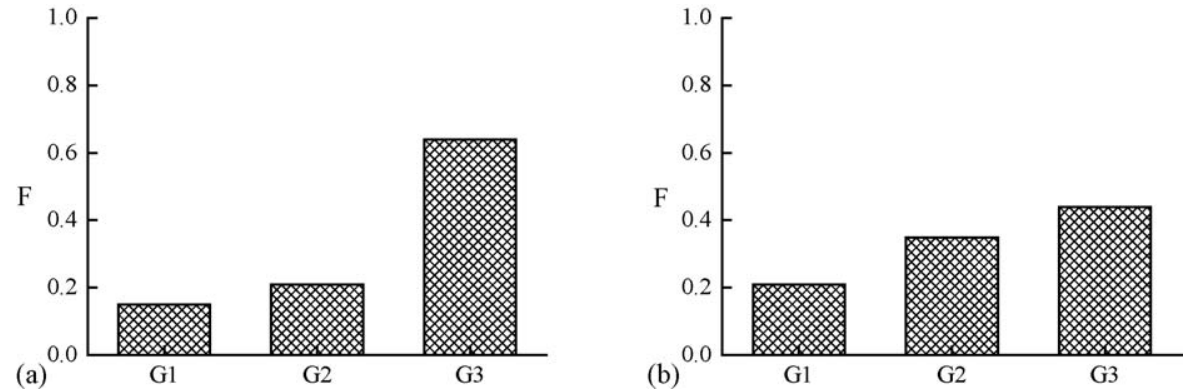

Fig. 7. Distribution of different kind of microstructure in tension after rolling, when tensile direction is normal (a) and parallel (b) to the previous rolling direction: G1—grains showing two sets of intersected dislocation walls; G2—grains showing equiaxed cells; G3—grains showing special microband features. 


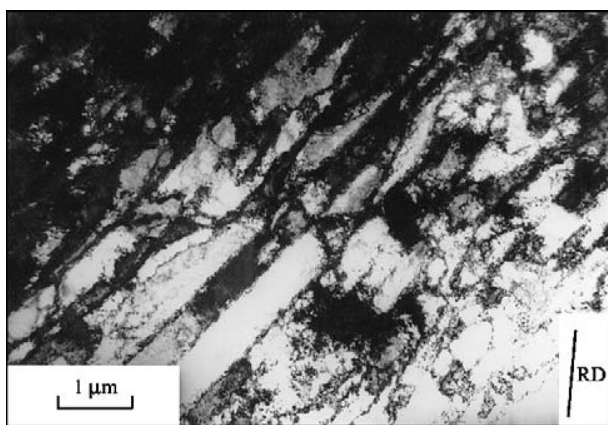

(a)

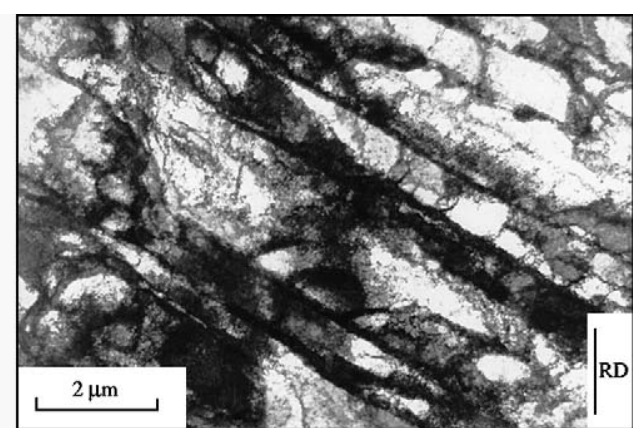

(b)

Fig. 8. Dislocation walls developed in the sequence tension (25\%)-rolling (10\%), rolling is normal to tension (RD: rolling direction).

path sequences performed on large grain polycrystals $(250 \mu \mathrm{m})$ [7] and on multicrystals reloaded in tension after prestraining in rolling, as in this study. In fact, the dislocation structure observed in this study, for simple tension, indicates the activation of only one main slip system for this strain path, which agrees with the fact that the strain accommodation processes between grains are negligible for large grain sizes, as discussed above. According to our knowledge, microbands appear after changing strain path as a result of interaction between a new active slip system and an "alien" dislocation microstructure resulting from the previous deformation, such as in case of severe changes of strain path: $\alpha$ is equal to zero [7]. This occurs for tension after normal rolling, which corresponds to a drastic change of strain path. For a smooth change of strain path such as tension after parallel rolling, the activation of new slip systems, not active during prestrain in rolling, is not expected in most grain orientations. The present results suggest that the avalanche glide, which takes place in the microbands, can occur on a slip system which has already been active during the first path. Thus, in the presence of a previously formed dislocation structure, the unique condition for the appearance of microbanding is glide on just one slip plane. This does not occur when multiple slip is imposed, as for the case of deformation of small grained samples, for which isolated microbands were not observed for the same cases of strain path change because of the strain accommodation process between grains [1-3].

For the tension-rolling sequence, we first analysed the case where the rolling direction is normal to the previous tensile axis. One or two families of long dislocation walls were observed in the samples subjected to 5 and $10 \%$ of rolling deformation after a tensile prestrain equal to $25 \%$ (Fig. 8). Rectangular cells, formed by intersecting dislocation walls, range from 0.4 to $0.9 \mu \mathrm{m}$ in size. These dislocation structures, typical of the second path in rolling, are roughly developed. For the sequence tension-normal rolling, a considerable number of grains still show isolated microbands (Fig. 9). These microbands lie in the $\left\{\begin{array}{lll}1 & 1 & 1\end{array}\right\}$ slip plane and were $0.1-0.3 \mu \mathrm{m}$ thick. These special microband features are produced by the appearance and development of new slip systems, which were latent during the first strain path in tension. In fact, orthogonal strain path change in the tension-rolling sequence is favourable to the activation of latent slip systems. Moreover, considering the large-grained material studied, some grains can deform in rolling by slip on just one system, as was also observed in simple rolling. This can explain the distribution of dislocation structures arising during the second strain path in rolling, when the rolling direction is normal to the previous tension, as is shown in Fig. 10a (the dislocation microstructure of more than 40 grains was studied), i.e. the relatively high density of isolated microbands observed for this strain path.

For the tension-rolling sequence, rolling being parallel to previous tension, the copper multicrystalline samples were prestrained up to $25 \%$ in tension and then reloaded up to 10 and $20 \%$ of deformation in rolling. During the second strain path in rolling, TEM observations showed three typical microstructures, as it was observed for rolling deformation normal to previous tensile prestrain, illustrated for the case of $20 \%$ of deformation in rolling: one and two sets of straight dislocation walls (Fig. 11) and isolated microbands (Fig. 12). These microbands lie in the

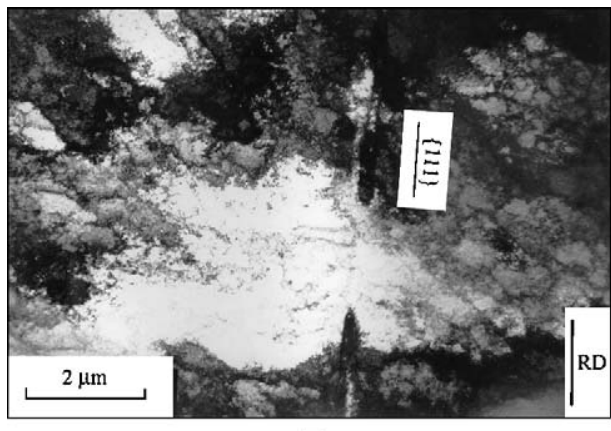

(a)

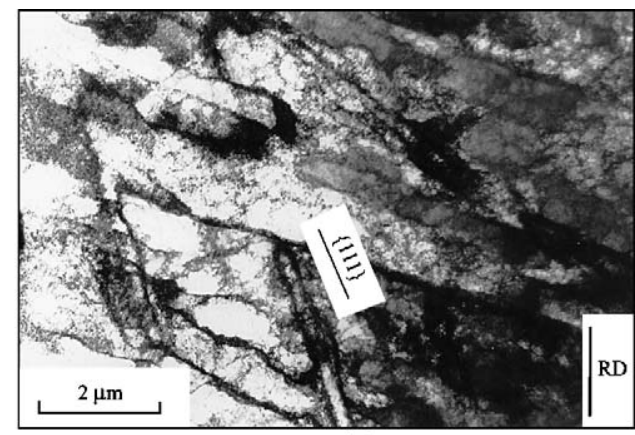

(b)

Fig. 9. Microbands developed in the sequence tension (25\%)-rolling (10\%), rolling is normal to tension (RD: rolling direction). 

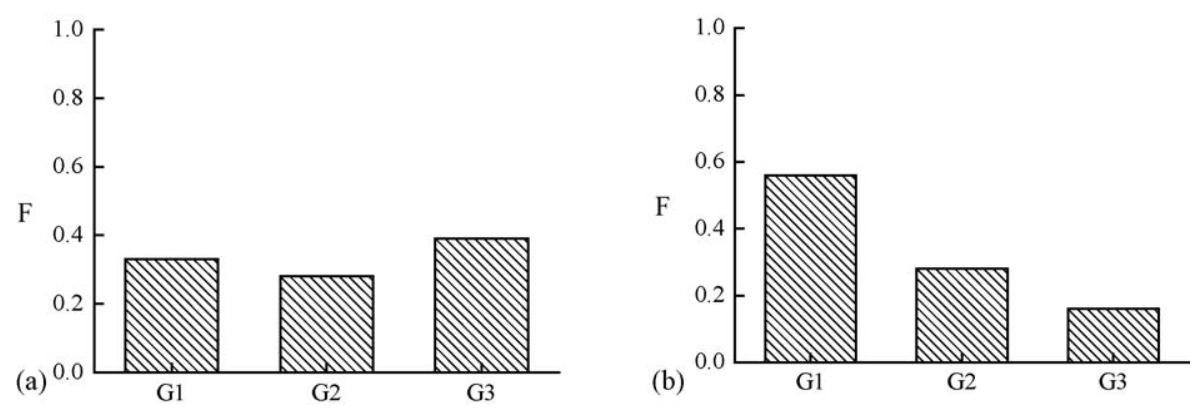

Fig. 10. Distribution of different kind of microstructure in rolling after tension, when rolling direction is normal (a) and parallel (b) to the previous tensile axis: G1—grains showing two sets of straight dislocation walls; G2 — grains showing one set of parallel dislocation walls; G3—grains showing special microband features.

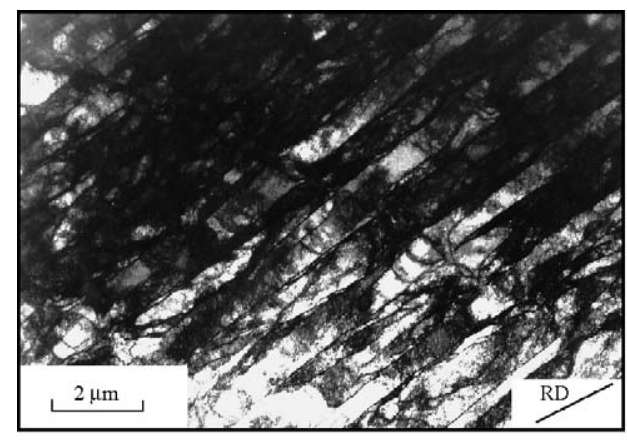

(a)

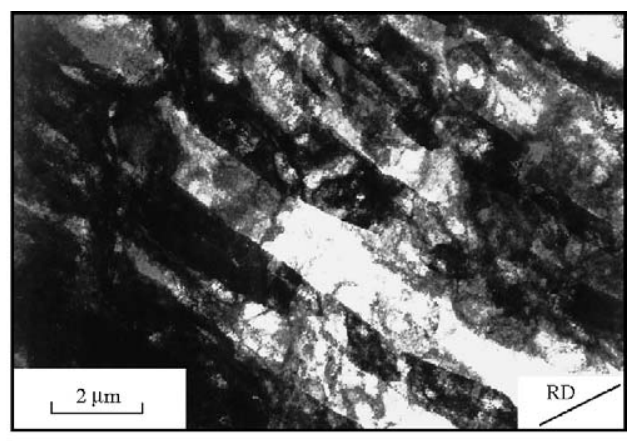

(b)

Fig. 11. Dislocation walls developed in the sequence tension (25\%)-rolling (20\%), rolling is parallel to tension (RD: rolling direction).

$\left\{\begin{array}{lll}1 & 1 & 1\end{array}\right.$ plane. The number of grains showing microbands is not significant for this strain path sequence. Therefore, the localization of deformation in microbands is not so important in the tension-rolling sequence, when the rolling direction is parallel to previous tensile direction. This can be understood taking into account that: (i) multiple slip can be imposed, in some grains, to accommodate grain deformation to the strain path in rolling, as discussed above for simple rolling (nevertheless, considering the large grain size material studied, other grains can deform in rolling by slip in just one system, as was also observed in simple rolling); (ii) when rolling is parallel to previous tension, it is expected that the active slip systems after reloading in rolling were already active during previous tension, in most grains. The simultaneous occurrence of both these conditions can explain the relatively low frequency of microband observation for the parallel tension-rolling sequence, as shown in
Fig. 10b. This figure concerns the observation of more than 40 grains.

\subsection{Work-hardening behaviour}

The work-hardening behaviour after path change was studied in tension after $20 \%$ of prestrain in rolling, in order to compare the response after the two different types of path changes under investigation: tensile axis parallel (smooth path change: $\alpha$ is equal to 0.87 ) and normal (drastic path change: $\alpha$ is equal to zero) to the rolling direction. The true stress $\sigma$-logarithmic strain $\varepsilon$ curves obtained allow us to plot the evolution of the work hardening rate $\theta=\mathrm{d} \sigma / \mathrm{d} \varepsilon$ as a function of the true stress $\sigma$, as shown in Fig. 13, for normal and parallel tension after prestrain in rolling. For both cases of strain path change, a sharp initial drop of the work hardening rate was observed, after which

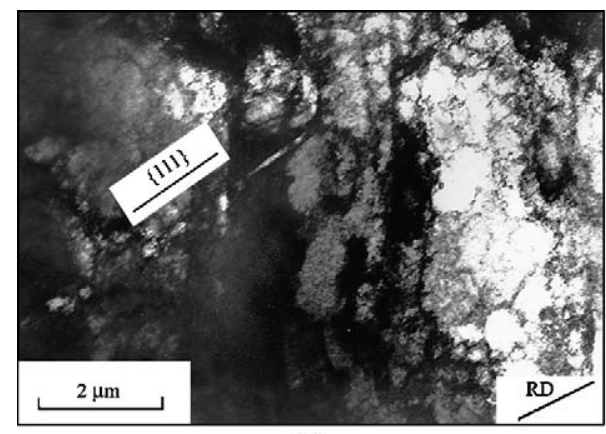

(a)

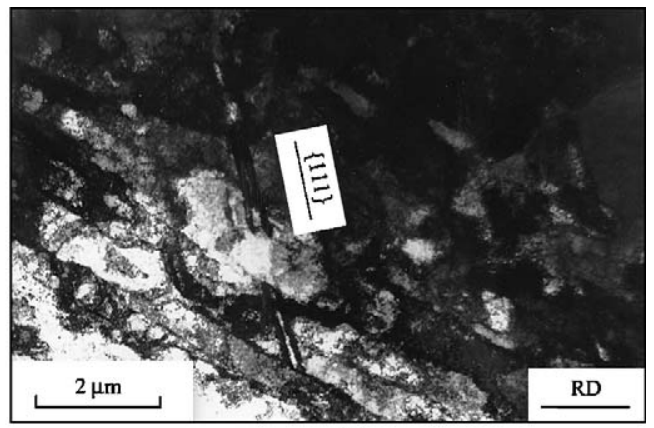

(b)

Fig. 12. Microbands developed in the sequence tension (25\%)-rolling (20\%), rolling is parallel to tension (RD: rolling direction). 


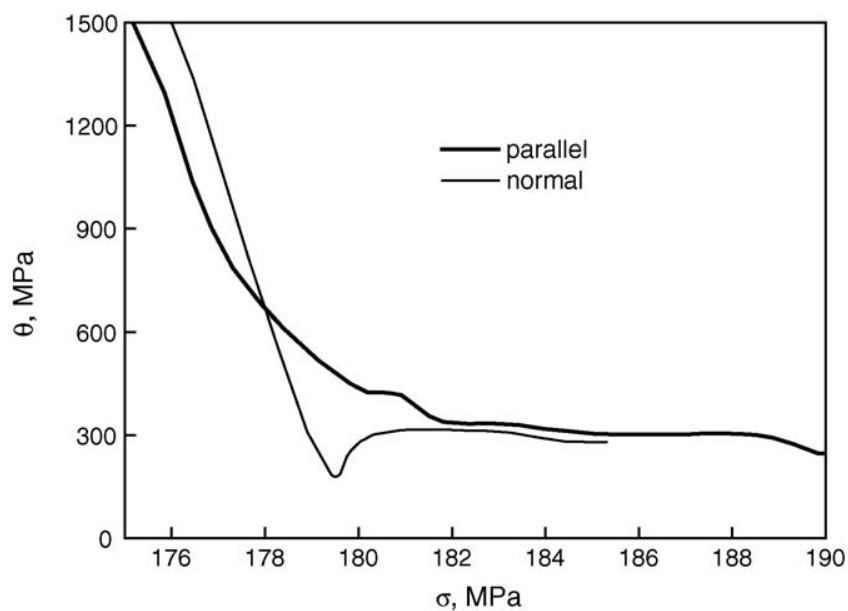

Fig. 13. Work-hardening rate $\theta=\mathrm{d} \sigma / \mathrm{d} \varepsilon$ vs. tensile stress $\sigma$, after normal and parallel strain path changes, for the rolling-tension sequence.

the copper samples reloaded in tension normal to previous rolling cross the line corresponding to tension parallel to rolling and reach their lowest points for a stress value slightly lower than $180 \mathrm{MPa}$. After this, the work hardening rate increases and stabilizes at a value close to $300 \mathrm{MPa}$, as for the case of tension parallel to previous rolling. This result means a stronger recovery in tension after normal rolling than after parallel rolling and agrees with the observed reorganization of the dislocation structure observed by TEM, after path change as discussed above. In fact, the activation of new slip systems after path change causes the transient observed in the work-hardening behaviour after normal path change, which is concomitant with the disappearance of some dislocation walls developed during prestrain, as observed previously in copper sheets with low and medium grain sizes [2,3]. This effect is less important after parallel path change: for this case one of the active slip systems during rolling must be active during the subsequent tension, in most of the grains. The work-hardening results from this study must be related to the much greater frequency of isolated microbands observed in tension after normal rolling compared to that of parallel rolling: the stronger recovery of the previous structure seems to be linked to the appearance of isolated microbands.

\section{Conclusions}

TEM analyses of the dislocation microstructure of copper multicrystalline sheets subjected to tension, rolling, tension after rolling and rolling after tension deformation sequences were carried out. The most common dislocation feature observed in simple tension was one family of straight parallel dislocation walls. This result points out the activation of only one slip system in most of grains, such as for single crystal deformation.
During simple rolling, the constraints imposed by this sequence give rise to the activation of two or more main slip systems in most grains, such as for fine-grained copper $(20 \mu \mathrm{m})$ deformed under the same path. In both tension-rolling and rolling-tension sequences isolated microbands have been observed after strain path change, although the observation frequency was higher for the rolling-tension sequence, when the tensile axis is normal to the rolling direction. These special microband features, developed after the change in strain path, whatever the sequence and the type of path change, and occur because of the specific properties of multicrystalline samples which have an additional degree of freedom in the direction perpendicular to the sample surface. Due to this fact, the strain accommodation processes between grains are almost negligible and lead to the localization of deformation in microbands. The microbands arise after changing strain path, because of the activation of only one new slip system, which promotes intensive glide in localized areas. This active slip system, which causes intense glide in the microband, may or may not be active during the previous strain path.

\section{Acknowledgements}

The authors are grateful to the Portuguese Foundation for Science and Technology (FCT) who financially supported this work, through the Program POCTI (Portuguese Government and FEDER). One of the authors, N.A. Sakharova, was supported by a grant for scientific research from the Portuguese Science and Technology Foundation. This support is gratefully acknowledged.

\section{References}

[1] J.V. Fernandes, M.F. Vieira, Metall. Mater. Trans. A 28 (1997) 1169.

[2] J.J. Grácio, J.V. Fernandes, J.H. Schmitt, Mater. Sci. Eng. A 118 (1989) 97.

[3] J.V. Fernandes, J.J. Gracio, J.H. Schmitt, in: C. Teodosiu, J.L. Raphanel, F. Sidoroff (Eds.), Large Plastic Deformations: Fundamental Aspects and Applications to Metal Forming, A.A. Balkema, Rotterdam, 1993, p. 219.

[4] P. Pinheiro, W.A. Monteiro, R. Barbosa, P.R. Cetlin, Mater. Sci. Eng. A 368 (2004) 280.

[5] X. Huang, N. Hansen, Mater. Sci. Eng. A 387-389 (2004) 186.

[6] I.P. Pinheiro, R. Barbosa, P.R. Cetlin, Scripta Mater. 44 (2001) 187.

[7] J.V. Fernandes, J.J. Gracio, J.H. Schmitt, E.F. Rauch, Scripta Metall. Mater. 28 (1993) 1335.

[8] E.C.S. Correa, M.T.P. Aguilar, P.R. Cetlin, J. Mater. Proc. Technol. 124 (2002) 384

[9] T. Leffers, V.S. Ananthan, H. Christoffersen, Mater. Sci. Eng. A 319-321 (2001) 148.

[10] V.V. Rybin, Bolshie Plasticheskie Deformatsii i Razrushenie Metallov, Metallurgiya, Moscow, 1986.

[11] J.H. Schmitt, E.L. Shen, J.L. Raphanel, Int. J. Plasticity 10 (1994) 535.

[12] J.H. Schmitt, J.V. Fernandes, J.J. Gracio, M.F. Vieira, Mater. Sci. Eng. A 147 (1991) 143. 